\title{
Unsteady free Convection and Mass Transfer Flow of Micropolar Fluid Embedded in a Porous Media
}

\author{
${ }^{1} \mathrm{H}$. Usman \\ 1 Department of Mathematics, \\ Usmanu Danfodiyo University, \\ Sokoto, Nigeria
}

\author{
${ }^{1}$ I. J. Uwanta \\ ${ }^{1}$ Department of Mathematics, \\ Usmanu Danfodiyo University, \\ Sokoto, Nigeria
}

\author{
${ }^{2}$ A.B. Baffa \\ ${ }^{2}$ Department of Mathematical \\ Science, Bayero University, \\ Kano, Nigeria
}

\begin{abstract}
This paper deals with the unsteady free convection and mass transfer flow of micropolar fluid embedded in a porous media. The governing equations involve the fluid and micropolar velocities respectively, temperature and concentration fields. The effects of material parameters on the velocities, temperature and concentration are discussed. Perturbation method is used to obtain the solutions to the governing equations. Results show that the velocity increases with an increase in Grashof and modified Grashof numbers $G$ and $\mathrm{Gm}$ respectively. While an increase in the Prandtl number $P r$ and Schmidt number Sc lead to a decrease in the temperature and the concentration respectively.
\end{abstract}

Keywords: Micropolar fluid, Grashof number, Modified Grashof number, Porous plate, Prandtl number.

\section{INTRODUCTION}

The study of flow and mass transfer for an electrically conducting micropolar fluid past a porous plate under the influence of a magnetic field has attracted the interest of many investigators in view of its applications in many engineering problems such as MagnetoHydroDynamic (MHD) generator, Oil exploration, Plasma studies, and Geothermal energy extractions. Soundalgekar and Takhar (1977). Micropolar fluids are fluids with micro structure belonging to a class of fluids with non-symmetrical stress tensor. Physically, they represent fluids consisting of randomly oriented particles suspended in viscous medium Aero et al (1965), Dep (1968) and Lukaszewicz (1999). Takhar and Agarwal (1998) studied the mixed convective flow of a steady, incompressible micropolar fluid over a stretching sheet. Kim (2001) studied the unsteady two-dimensional laminar flow of a viscous incompressible micropolar fluid past a semi-infinite porous plate embedded in a porous medium. Uwanta (2002) studied micropolar fluid flow in a channel with Poiseuille effects. Kim (2003) investigated transient mixed radiative convection flow of a micropolar fluid past a moving, semi infinite vertical porous plate. Hassanien and Essawy (2004) studied the natural convection flow of micropolar fluid from a permeable uniform heat flux surface in porous media. Makinde and Mhone (2005) have investigated heat transfer to MHD oscillatory flow in a channel filled with porous medium. Lok et al (2006) investigated unsteady mixed convection flow of a micropolar fluid near the stagnation point on a vertical surface. Uwanta (2008) studied the effects of mass transfer on laminar convective hydromagnetic flow of radiating gas in a vertical infinite channel. Mostafa (2009) studied thermal radiation effect on unsteady MHD free convection flow past a vertical plate with temperature dependent viscosity. Ahmad (2010) has studied the effects of thermophoresis on natural convection boundary layer flow of a micropolar fluid.

\section{GOVERNING EQUATIONS}

In Cartesian coordinate system, we consider the twodimensional unsteady flow of a laminar incompressible micropolar fluid with heat and mass transfer in the presence of chemical reaction past a vertical porous moving plate embedded in a porous medium and subjected to a transverse magnetic field in the presence of a pressure gradient. The analysis is based on the assumption that the viscous and Darcy resistance terms are taken into account with constant permeability porous medium. Under these conditions, the governing equations for the problem are

Continuity equation:

$$
\frac{\partial L^{*}}{\partial *^{*}}=0
$$

Linear momentum equation:

$$
\begin{aligned}
& \frac{\partial U^{*}}{\partial^{*}}+V^{*} \frac{\partial U^{*}}{\partial y^{*}}=\frac{1}{\rho} \frac{\partial^{*}}{\partial x^{*}}\left(v+V_{r}\right) \frac{\partial^{2} U^{*}}{\partial *^{*}}+g \beta_{f}\left(T-T_{\infty}\right)- \\
& v^{U^{*}}+2 V_{r} \frac{\partial w^{*}}{\partial y^{*}}+g \beta_{c}\left(C-C_{\infty}\right)
\end{aligned}
$$

Angular momentum equation:

$\rho j^{*}\left(\frac{\partial w^{*}}{\partial t^{*}}+V^{*} \frac{\partial w^{*}}{\partial y^{*}}\right)=\gamma \frac{\partial^{2} w^{*}}{\partial y^{* 2}}$

Energy equation:

$\frac{\partial T}{\partial t^{*}}+V^{*} \frac{\partial T}{\partial y^{*}}=\alpha \frac{\partial^{2} T}{\partial y^{* 2}}$

Diffusion equation:

$\frac{\partial C^{*}}{\partial t^{*}}+V^{*} \frac{\partial C^{*}}{\partial y^{*}}=D^{*} \frac{\partial^{2} C^{*}}{\partial y^{* 2}}-K^{*} C^{*}$

where $x^{*}$ and $y^{*}$ are the dimensional distances along and perpendicular to the plate respectively, $U^{*}, V^{*}$ are the components of dimensional velocities along $x^{*}$ and $y^{*}$ respectively, $\rho$ is the fluid density, $v$ is the fluid kinematic viscosity, $V_{r}$ is the fluid kinematic rotational viscosity, $g$ is the acceleration due to gravity, $\beta_{f}$ and $\beta_{c}$ are the coefficients of volume expansions for temperature and concentration, $K^{*}$ is an empirical constant called permeability of the porous medium, $j^{*}$ is the micro-inertia density, $w^{*}$ is the component of the angular velocity, $\gamma$ is the spin-gradient 
viscosity, $T$ is the temperature, $C^{*}$ is the component of dimensional concentration, $\alpha$ is the fluid thermal diffusivity, $D$ is the coefficient of mass diffusivity. The first term on the RHS of (2.2) is the pressure term, the second term is the viscous term, the third term is the buoyancy due to temperature difference, the fourth term is the Darcy or porous term, the fifth term is the micropolar term while the last is the mass term.

The boundary conditions for the velocities, temperature and concentration are

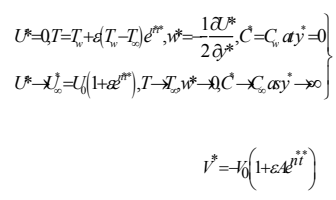

Where $n^{*}$ is the dimensionless exponential index, $U_{\infty}{ }^{*}$ is the free streamvelocity, $U_{0}$ is a scale of free stream velocity, $A$ is a real positive constant of suction velocity parameter, $\varepsilon$ and $\varepsilon A$ are small less than unity, i.e. $\varepsilon A<<1, V_{0}$ is a scale of suction velocity normal to the plate and is assumed as a function of time only.

Outside the boundary layer, the pressure term in (2.2) gives $-\frac{1}{\rho} \frac{d p^{*}}{d x^{*}}=\frac{d U_{\infty}^{*}}{d t^{*}}+\frac{v}{k^{*}} U_{\infty}^{*}$

We now introduce the following dimensionless variables as follows:

$$
\left.\begin{array}{l}
U=\frac{U^{*}}{U_{0}}, V=\frac{v^{*}}{V_{0}}, y=\frac{V_{0} y^{*}}{v}, U_{\infty}=\frac{U_{\infty}^{*}}{U_{0}}, w=\frac{v}{U_{0} V_{0}} w^{*}, t=\frac{t^{*} V_{0}^{2}}{v}, \\
\theta=\frac{T-T_{\infty}}{T_{w}-T_{\infty}}, n=\frac{n^{*} v}{V_{0}^{2}}, K=\frac{k^{*} V_{0}^{2}}{V^{2}}, j=\frac{V_{0}^{2}}{v^{2}} J^{*}, S_{c}=\frac{v}{D^{*}}, \mathrm{Pr}=\frac{v}{\alpha}, \\
G=\frac{\nu_{f} g\left(T_{w}-T_{\infty}\right)}{U_{0} V_{0}^{2}}, G m=\frac{y_{f} g\left(C_{w}-C_{\infty}\right)}{U_{0} V_{0}^{2}}, \gamma=u j^{*}\left(1+\frac{1}{2} \beta\right), \beta=\frac{\Lambda}{\mu}, C=\frac{C^{*}-C_{\infty}}{C_{w}-C_{\infty}}
\end{array}\right\}
$$

Where $\gamma$ is the spin-gradient viscosity, $\beta$ is the dimensionless viscosity ratio and $\Lambda$ is the coefficient of gyro-viscosity or vortex viscosity.

In view of equation (2.9), the governing equations (2.2) (2.6) reduce to the following non-dimensional form:

$$
\begin{aligned}
& \frac{\partial U}{\partial t}-\left(1+\varepsilon A e^{n t}\right) \frac{\partial U}{\partial y}=\frac{d U_{\infty}}{d t}+(1+\beta) \frac{\partial^{2} U}{\partial y^{2}}+G \theta+\frac{1}{K}\left(U_{\infty}-U\right)+2 \beta \frac{\partial w}{\partial y}+G m C \\
& \frac{\partial w}{\partial t}-\left(1+\varepsilon A e^{n t}\right) \frac{\partial w}{\partial y}=\frac{1}{\eta} \frac{\partial^{2} w}{\partial y^{2}} \\
& \frac{\partial \theta}{\partial t}-\left(1+\varepsilon A e^{n t}\right) \frac{\partial \theta}{\partial y}=\frac{1}{\operatorname{Pr}} \frac{\partial^{2} \theta}{\partial y^{2}} \\
& \frac{\partial C}{\partial t}-\left(1+\varepsilon A e^{n t}\right) \frac{\partial C}{\partial y}=\frac{1}{S_{c}} \frac{\partial^{2} C}{\partial y^{2}}-K C
\end{aligned}
$$

Where $\beta=\frac{V_{r}}{V}, \eta=\frac{\mu j^{*}}{\gamma}, \operatorname{Pr}$ is the Prandtl number, $\mathrm{G}$ is the Grashof number, $\mathrm{Gm}$ is the modified Grashof number and $\mathrm{Sc}$ is the Schmidt number.

The boundary conditions (2.6) are given by the following dimensionless form:

$$
\left.\begin{array}{l}
U=0, \theta=1+\varepsilon e^{n t}, w=-\frac{1}{2} \frac{\partial U}{\partial y}, C=1 \text { on } y=0 \\
U \rightarrow U_{\infty}, \theta \rightarrow 0, w \rightarrow 0, C \rightarrow 0 \text { as } y \rightarrow \infty
\end{array}\right\}
$$

In order to reduce the above system of partial differential equations to a system of ordinary differential equations in dimensionless form, we represent the linear, angular velocities, temperature and mass as:

$$
\begin{aligned}
& U(y, t)=U_{0}(y)+\varepsilon e^{n t} U_{1}(y)+O\left(\varepsilon^{2}\right) \\
& w(y, t)=w_{0}(y)+\varepsilon e^{n t} w_{1}(y)+O\left(\varepsilon^{2}\right) \\
& \theta(y, t)=\theta_{0}(y)+\varepsilon e^{n t} \theta_{1}(y)+O\left(\varepsilon^{2}\right) \\
& C(y, t)=C_{0}(y)+\varepsilon e^{n t} C_{1}(y)+O\left(\varepsilon^{2}\right)
\end{aligned}
$$

Substituting equations (2.15) - (2.18) into equations (2.10) (2.14) and neglecting the coefficient of higher order terms reduce to the zeroth and first orders respectively:

$$
\begin{gathered}
(1+\beta) U_{0}^{\prime \prime}+U_{0}^{\prime}-\frac{1}{k} U_{0}=-\frac{1}{k}-G \theta_{0}-G m C_{0}-2 \beta w_{0}^{\prime} \\
w_{0}^{\prime \prime}+\eta w_{0}^{\prime}=0 \\
\theta_{0}^{\prime \prime}+\operatorname{Pr} \theta_{0}^{\prime}=0 \\
C_{0}^{\prime \prime}+S_{c} C_{0}^{\prime}-S_{c} k^{2} C_{0}=0
\end{gathered}
$$

subject to the following boundary conditions:

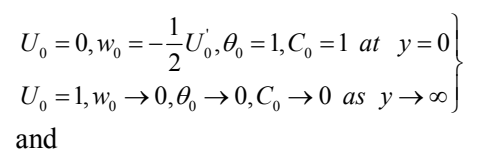

$$
\begin{aligned}
& (1+\beta) U_{1}^{\prime \prime}+U_{1}^{\prime}-\left(\frac{1}{k}+n\right) U_{1}=-\left(\frac{1}{k}+n\right)-A U_{0}^{\prime}-G \theta_{1}-G m C_{1}-2 \beta w_{1}^{\prime} \\
& w_{1}^{\prime \prime}+\eta w_{1}^{\prime}-n \eta w_{1}=-A \eta w_{0}^{\prime} \\
& \theta_{1}^{\prime \prime}+\operatorname{Pr} \theta_{1}^{\prime}-n \operatorname{Pr} \theta_{1}=-A \operatorname{Pr} \theta_{0}^{\prime} \\
& C_{1}^{\prime \prime}+S_{c} C_{1}^{\prime}-\left(K^{2}+n\right) S_{c} C_{1}=-A S_{c} C_{0}^{\prime}
\end{aligned}
$$

subject to the following boundary conditions

$$
\left.\begin{array}{l}
U_{1}=0, w_{1}=-\frac{1}{2} U_{1}^{\prime}, \theta_{1}=1, C_{1}=0 \text { at } y=0 \\
U_{1}=1, w_{1} \rightarrow 0, \theta_{1} \rightarrow 0, C_{1} \rightarrow 0 \text { as } y \rightarrow \infty
\end{array}\right\}
$$

The solutions of equations (2.19) - (2.22), (2.24) - (2.27) subject to the boundary conditions (2.23) and (2.28) are respectively:

$$
\begin{aligned}
& U_{0}(y)=1+a_{1} e^{-h_{2} y}+a_{2} e^{-\operatorname{Pr} y}+a_{3} e^{-r y}+a_{4} e^{-\eta \eta y} \\
& U_{1}(y)=1+b_{1} e^{-h_{4} y}+b_{2} e^{-h_{2} y}+b_{3} e^{-\lambda_{2} y}+b_{4} e^{-F_{2} y}+b_{5} e^{-k_{2} y}+b_{6} e^{-\mathrm{Pr} y}+b_{7} e^{-r y}+b_{8} e^{-\eta y} \\
& w_{0}(y)=c_{1} e^{-\eta y y} \\
& w_{1}(y)=c_{2} e^{-F_{2} y}-\frac{A}{n} \eta c_{1} e^{-\eta \eta y} \\
& \theta_{0}(y)=e^{-\operatorname{Pr} y} \\
& \theta_{1}(y)=\left(1+\frac{A}{n} \operatorname{Pr}\right) e^{-k_{2} y}-\frac{A}{n} \operatorname{Pr} e^{-\operatorname{Pr} y} \\
& C_{0}(y)=e^{-r y}
\end{aligned}
$$


$C_{1}(y)=B *\left(e^{-r y}-e^{-\lambda_{2} y}\right)$

where

$$
\begin{aligned}
& h_{2}=\frac{1}{2(1+\beta)}\left[1+\sqrt{1+\frac{4}{k}(1+\beta)}\right], \quad h_{4}=\frac{1}{2(1+\beta)}\left[1+\sqrt{1+4(1+\beta)\left(\frac{1}{k}+n\right)}\right] \\
& \lambda_{2}=\frac{1}{2}\left[S_{c}+\sqrt{S_{c}^{2}+4\left(k^{2}+n\right) S_{c}}\right], \quad F_{2}=\frac{\eta}{2}\left[1+\sqrt{1+\frac{4 n}{\eta}}\right], \quad k_{2}=\frac{\operatorname{Pr}}{2}\left[1+\sqrt{1+\frac{4 n}{\operatorname{Pr}}}\right], \\
& r=\frac{1}{2}\left[S_{c}+\sqrt{S_{c}^{2}+4 k^{2} S_{c}}\right], \quad B^{*}=\frac{r A S_{c}}{r^{2}-r S_{c}-\left(k^{2}+n\right) S_{c}}, a_{1}=-1-a_{2}-a_{3}-a_{4}, \\
& a_{2}=-\frac{G}{(1+\beta) \operatorname{Pr}^{2}-\operatorname{Pr}-\frac{1}{k}}, a_{3}=-\frac{G m}{(1+\beta) r^{2}-r-\frac{1}{k}}{ }^{\prime} a_{4}=\frac{2 \beta \eta c_{1}}{(1+\beta) \eta^{2}-\eta-\frac{1}{k}} \\
& b_{1}=-1-b_{2}-b_{3}-b_{4}-b_{5}-b_{6}-b_{7}-b_{8}, \quad b_{2}=\frac{A a_{1} h_{2}}{(1+\beta) h_{2}^{2}-h^{2}-\left(\frac{1}{k}+n\right)}, \\
& b_{3}=\frac{G m B^{*}}{(1+\beta) \lambda_{2}^{2}-\lambda_{2}-\left(\frac{1}{k}+n\right)} b^{\prime} b_{4}=\frac{2 \beta F_{2} c_{2}}{(1+\beta) F_{2}^{2}-F_{2}-\left(\frac{1}{k}+n\right)}, \\
& b_{5}=\frac{-G\left(1+\frac{A}{n} \operatorname{Pr}\right)}{(1+\beta) k_{2}^{2}-k_{2}-\left(\frac{1}{k}+n\right)}, b_{6}=\frac{A \operatorname{Pr}\left(\frac{G}{n}+a_{2}\right)}{(1+\beta) \operatorname{Pr}^{2}-\operatorname{Pr}-\left(\frac{1}{k}+n\right)}, \\
& b_{7}=\frac{r A a_{3}-G m B^{*}}{(1+\beta) r^{2}-r-\left(\frac{1}{k}+n\right)} b_{8}=\frac{A \eta\left(a_{4}-2 \frac{\beta \eta}{n} c_{1}\right)}{(1+\beta) \eta^{2}-\eta-\left(\frac{1}{k}+n\right)} \\
& c_{1}=\frac{k_{3} k_{4}}{2\left(k_{3}-\eta^{2} \beta\right)}, c_{2}=\frac{k_{1}\left(A \frac{\eta}{n} c_{1}+\frac{1}{2} k_{2}\right)}{k_{1}-F_{2}^{2} \beta}, k_{1}=(1+\beta) F_{2}^{2}-F_{2}-\left(\frac{1}{k}+n\right), \\
& k_{5}=b_{1} h_{4}+b_{2} h_{2}+b_{3} \lambda_{2}+b_{5} k_{2}+b_{6} \operatorname{Pr}+b_{7} r+b_{8} \eta \\
& k_{3}=(1+\beta) \eta^{2}-\eta-\frac{1}{k}, k_{4}=a_{1} h_{2}+a_{2} \operatorname{Pr}+a_{3} r
\end{aligned}
$$

By virtue of equations (2.15) - (2.18), we obtain the streamwise, angular velocities, temperature and mass transfer as follows:

$$
\begin{aligned}
& U(y, t)=1+a_{1} e^{-h_{2} y}+a_{2} e^{-\mathrm{Pr}_{r} y}+a_{3} e^{-\eta y}+a_{4} e^{-\eta y y}+\varepsilon e^{n t} \\
& \quad\left[1+b_{1} e^{-h_{4} y}+b_{2} e^{-h_{2} y}+b_{3} e^{-\lambda_{2} y}+b_{4} e^{-F_{2} y}+b_{5} e^{-k_{2} y}+b_{6} e^{-\mathrm{Pr}_{r} y}+b_{7} e^{-r y}+b_{8} e^{-\eta y y}\right] \\
& w(y, t)=c_{1} e^{-\eta \eta y}+\varepsilon e^{n t}\left[c_{2} e^{-F_{2} y}-\frac{A}{n} \eta c_{1} e^{-\eta_{y} y}\right] \\
& \theta(y, t)=e^{-\operatorname{Pr} y}+\varepsilon e^{n t}\left[e^{-k_{2} y}+\frac{A}{n} \operatorname{Pr}\left(e^{-k_{2} y}-e^{-\operatorname{Pr} y}\right)\right] \\
& C(y, t)=e^{-r y}+\varepsilon e^{n t}\left[B^{*}\left(e^{-r y}-e^{-\lambda_{2} y}\right)\right]
\end{aligned}
$$

\begin{tabular}{|c|c|c|c|c|}
\hline$y$ & $U$ & $w$ & $\theta$ & $c$ \\
\hline 0.0 & 0 & -1.586 & 1.0739 & 1 \\
\hline 0.1 & 0.2889 & -1.5006 & 0.9949 & 0.9654 \\
\hline 0.2 & 0.5273 & -1.4201 & 0.9221 & 0.9821 \\
\hline 0.3 & 0.7234 & -1.3443 & 0.8548 & 0.8999 \\
\hline 0.4 & 0.8839 & -1.2728 & 0.7927 & 0.8689 \\
\hline 0.5 & 10146 & -1.2053 & 0.7352 & 0.8389 \\
\hline 0.6 & 1.1203 & -1.1416 & 0.6822 & 0.81 \\
\hline 0.7 & 1.2052 & -1.0815 & 0.6331 & 0.7821 \\
\hline 0.8 & 1.2727 & $-1.024 B$ & 0.5876 & 0.7551 \\
\hline 0.9 & 1.3257 & -0.9712 & 0.5456 & 0.7291 \\
\hline 1.0 & 1.3666 & -0.9206 & 0.5066 & 0.7041 \\
\hline 1.1 & 1.3975 & -0.8727 & 0.4706 & 0.6798 \\
\hline 1.2 & 1.4202 & -0.8275 & 0.4372 & 0.6565 \\
\hline 1.3 & 1.436 & -0.7847 & 0.4062 & 0.6839 \\
\hline 1.4 & 1.4462 & -0.7442 & 0.3775 & 0.6121 \\
\hline 1.5 & 1.4517 & -0.7069 & 0.3509 & 0.5911 \\
\hline 1.6 & 1.4536 & -0.6697 & 0.3262 & 0.5708 \\
\hline 1.7 & 1.4525 & -0.6554 & 0.3035 & 0.5512 \\
\hline 1.8 & 1.4489 & -0.603 & 0.2821 & 0.5323 \\
\hline 1.9 & 1.4434 & -0.5722 & 0.2623 & 0.514 \\
\hline 2.0 & 1.4364 & -0.5431 & 0.244 & 0.4964 \\
\hline 2.1 & 1.4283 & -0.5155 & 0.227 & 0.4793 \\
\hline 2.2 & 1.4193 & -0.4894 & 0.2112 & 0.4629 \\
\hline 2.3 & 1.4096 & -0.4646 & 0.1965 & 0.4471 \\
\hline 2.4 & 1.3996 & -0.4412 & 0.1829 & 0.4317 \\
\hline 2.5 & 1.3892 & -0.4189 & 0.1702 & 0.4169 \\
\hline 2.6 & 1.3787 & -0.3978 & 0.1584 & 0.4027 \\
\hline 2.7 & 1.3682 & -0.3778 & 0.1475 & 0.3889 \\
\hline 2.8 & 1.3577 & -0.3589 & 0.1373 & 0.3756 \\
\hline 2.9 & 1.3473 & -0.3409 & 0.1278 & 0.3627 \\
\hline 3.0 & 1.337 & -0.3238 & 0.119 & 0.3503 \\
\hline 3.1 & 1.327 & -0.3076 & 0.1108 & 0.3383 \\
\hline 3.2 & 1.3172 & -0.2923 & 0.1032 & 0.3268 \\
\hline 3.3 & 1.3077 & -0.2777 & 0.0961 & 0.3156 \\
\hline 3.4 & 1.2985 & -0.2688 & 0.0895 & 0.3048 \\
\hline 3.5 & 1.2895 & -0.2507 & 0.0834 & 0.2944 \\
\hline 3.6 & 1.2809 & -0.2382 & 0.0777 & 0.2843 \\
\hline 3.7 & 1.2725 & -0.2264 & 0.0723 & 0.2746 \\
\hline 3.8 & 1.2645 & -0.2151 & 0.0674 & 0.2652 \\
\hline 3.9 & 1.2568 & -0.2045 & 0.0628 & 0.2562 \\
\hline 4.0 & 1.2493 & -0.1943 & 0.0585 & 0.2474 \\
\hline
\end{tabular}

We can now calculate the Skin friction as follows:

$$
\begin{gathered}
\tau_{w}=\left.\frac{\partial u}{\partial y}\right|_{y=0}=-a_{1} h_{2}-a_{2} \operatorname{Pr}-a_{3} r-a_{4} \eta+\varepsilon e^{n t}\left[-b_{1} h_{4}-b_{2} h_{2}-b_{3} \lambda_{2}-b_{4} F_{2}\right. \\
\left.-b_{5} k_{2}-b_{6} \operatorname{Pr}-b_{7} r-b_{8} \eta\right]
\end{gathered}
$$

The heat transfer coefficient in terms of Nusselt number is as follows:

$$
N_{u}=\left.\frac{\partial \theta}{\partial y}\right|_{y=0}=-\operatorname{Pr}+\varepsilon e^{n t}\left[\frac{A}{n} \operatorname{Pr}^{2}-k_{2}\left(1+\frac{A}{n} \operatorname{Pr}\right)\right]
$$

\section{RESULTS AND DISCUSSION}

Values of streamwise velocity, angular velocity, temperature and mass transfer are presented in Table 1 where; $n=1, t=2, \varepsilon=0.01, G=1, G m=1, A=1, S c=0.2, \beta=0.4, \eta=0.5, \operatorname{Pr}=0.7$
Table 1: Showing various distributions

Values of stream wise velocity, angular velocity, temperature and mass transfer are presented in Table 2 where

$n=2, t=5, \varepsilon=0.07, G=5, G m=3, A=1, S c=0.2, \beta=0.4, \eta=0.5, \operatorname{Pr}=1$ 
Table 2: Showing various distributions.

\begin{tabular}{|c|c|c|c|c|}
\hline$y$ & $U(x 1 \infty 00)$ & $w(\triangle \triangle \infty 00)$ & $\theta(\times 1000)$ & c \\
\hline 0.0 & 0.0000 & -3.8394 & 1.5429 & 1.0000 \\
\hline 0.1 & 0.6465 & -3.2301 & 1.1969 & 9.9278 \\
\hline 0.2 & 10919 & -2.7928 & 0.9199 & $-19 . \oplus 95$ \\
\hline 0.3 & 1.3883 & -2.36066 & 0.6989 & -28.3268 \\
\hline 0.4 & 1.5752 & -1.9998 & 0.5231 & $-35 . \Phi_{14}$ \\
\hline 0.5 & 1.6830 & -1.6794 & 0.3838 & -42.7509 \\
\hline 0.6 & 17345 & -1.4014 & 0.2741 & -48 . 137 \\
\hline 0.7 & 1.7471 & -1.1605 & $0.18 B 0$ & -53.2526 \\
\hline 0.8 & 1.7336 & -0.9521 & 0.1210 & $-58 . .545$ \\
\hline 0.9 & 17035 & -0.7721 & 0.0693 & -62.2211 \\
\hline 1.0 & 1.6638 & -0.6170 & 0.0298 & -65.5189 \\
\hline 1.1 & 16194 & -0.4835 & 0.0000 & -68.3001 \\
\hline 1.2 & 1.5738 & -0.3690 & -0.0221 & -70.6124 \\
\hline 1.3 & 1.5294 & -0.2710 & -0.0381 & -72.497 \\
\hline 1.4 & 1.4877 & -0.1874 & -0.0492 & -74.0025 \\
\hline 1.5 & 1.4496 & -0.1164 & -0.0566 & -75.1578 \\
\hline 1.6 & 1.4157 & -0.0562 & -0.0612 & -75.995 \\
\hline 1.7 & 1.3861 & -0.0055 & -0.0635 & -76.569 \\
\hline 1.8 & 1.3609 & 0.0369 & -0.0641 & -76.2545 \\
\hline 1.9 & 1.3398 & 0.0722 & -0.0634 & -76.9425 \\
\hline 2.0 & 13225 & 0.1013 & -0.0618 & -76.8169 \\
\hline 2.1 & 1.3088 & 0.1251 & -0.0596 & -76.5096 \\
\hline 2.2 & 1.2983 & 0.1443 & -0.0569 & -76.0407 \\
\hline 2.3 & 1.2907 & 0.1595 & -0.0539 & $-75 . \triangle 2 B 6$ \\
\hline 2.4 & 1.2856 & 0.1712 & -0.0508 & $-74 . \infty \infty$ \\
\hline 2.5 & 1.2826 & 0.1801 & -0.0476 & -73.8408 \\
\hline 2.6 & 1.2815 & 0.1864 & -0.0444 & -72 : 394 \\
\hline 2.7 & 1.2821 & 0.1907 & -0.0413 & -71.2520 \\
\hline 2.8 & 1.2840 & 0.1931 & $-0.03 \mathrm{BB}$ & -70.7578 \\
\hline 2.9 & 1.2870 & 0.1940 & -0.0354 & -69.9913 \\
\hline 3.0 & 1.2909 & 0.1936 & -0.0326 & -68.3720 \\
\hline 3.1 & 1.2956 & 0.1921 & -0.0300 & -67.087 \\
\hline 3.2 & 1.3008 & 0.1898 & -0.0275 & -65.8098 \\
\hline 3.3 & 1.3066 & 0.1867 & -0.0253 & -64.4808 \\
\hline 3.4 & 13126 & 0.1830 & -0.0231 & -63.1298 \\
\hline 3.5 & 1.3190 & 0.1789 & -0.0211 & -61.7620 \\
\hline 3.6 & 1.3255 & 0.1743 & -0.0198 & $-60 . \mp 28$ \\
\hline 3.7 & 1.3821 & 0.1695 & -0.0176 & $-58 . \Phi 67$ \\
\hline 3.8 & 1.3887 & 0.1645 & -0.0161 & -57.60182 \\
\hline 3.9 & 1.3453 & 0.1594 & -0.0145 & -56.2210 \\
\hline 4.0 & 1.3519 & 0.1541 & -0.0133 & $-54 . \mathbb{8} 84$ \\
\hline
\end{tabular}

Table 3: Values of $\tau_{w}$ and $N_{u}$ with different values of material parameters.

\begin{tabular}{|c|c|c|c|c|c|c|c|c|c|}
\hline$A$ & $n$ & $t$ & $\varepsilon$ & $G$ & $\operatorname{Pr}$ & $G m$ & $S C$ & $r_{W}$ & $N_{I}$ \\
\hline 1 & 1 & 2 & 0.01 & 1 & 0.7 & 1 & 0.2 & 3.1720 & -0.8217 \\
\hline 1 & 1 & 5 & 0.01 & 1 & 0.7 & 1 & 0.2 & 7.7254 & -3.1440 \\
\hline 1 & 1 & 2 & 0.01 & 5 & 0.7 & 2 & 0.2 & 7.9039 & -0.8217 \\
\hline 1 & 1 & 5 & 0.01 & 5 & 0.7 & 3 & 0.2 & 15.3520 & -3.1440 \\
\hline 1 & 2 & 2 & 0.01 & 1 & 0.7 & 1 & 0.2 & 4.7967 & -1.7337 \\
\hline 1 & 2 & 5 & 0.01 & 5 & 0.7 & 1 & 0.2 & 1180.10 & -417.7183 \\
& & & & & & & & 00 & \\
\hline 1 & 2 & 5 & 0.07 & 1 & 1 & 2 & 0.2 & 5157.50 & \\
& & & & & & & & 00 & 3855.6000 \\
\hline 1 & 2 & 2 & 0.07 & 1 & 1 & 1 & 0.2 & 15.6310 & -10.5547 \\
\hline 1 & 1 & 2 & 0.07 & 1 & 1 & 1 & 0.2 & 4.4801 & -2.1566 \\
\hline 1 & 1 & 5 & 0.07 & 1 & 1 & 1 & 0.2 & 35.5149 & -24.2303 \\
\hline
\end{tabular}

Values of $U, w, \theta$ and $\mathrm{C}$ are presented in Table 1, where $n=1, A=1, t=2, \varepsilon=0.01$,

$, \eta=0.5, S_{c}=0.2, \operatorname{Pr}=0.7, G=1, G m=1, \beta=0.4 . \quad$ For the reactive flow, the velocity shows high values reaching a maximum of 1.4525 while the angular velocity is negative. Also temperature field and mass transfer are high at the origin.

In Table 2, values of $U, w, \theta$ and $C$ are presented while other constants are as before. For the reactive flow, the velocity profile shows high values reaching a maximum of 1747.1, while angular velocity is still negative. Temperature field and mass transfer are high at the origin.

The effect of the material parameters $n, t, \varepsilon, G, G m$ and Pron the streamwise velocity and angular velocity past a vertical plate are presented in Tables 1 and 2. The analytical results show that the value of the velocity distribution across the boundary layer is higher with an increase in the material parameters as compared with a micropolar fluid. In addition the value of the angular velocity on the porous plate decreases as the material parameters are increased.

Typical variation of the temperature fields along the $y$ coordinate are shown in Tables 1 and 2 respectively for different values of Prandtl number Pr. The analytical results shows that an increase of Prandtl number results in a decreasing thermal boundary layer thickness and more uniform temperature distribution across the boundary layer. The reason is that smaller values of $\operatorname{Pr}$ are equivalent to increasing thermal conductivities and therefore heat is able to diffuse away from the heated surface more rapidly than for higher values of Pr. Hence the boundary layer is thicker and 
the rate of heat transfer is reduced, for gradients have been reduced.

The variations of the mass transfer along the y-coordinate are presented in Tables 1 and 2. The analytical results show that an increase in the dimensionless exponential index $n$ and $t$ results to a decrease in the values of mass transfer.

The variation of the surface skin friction $\tau_{w}$ on the porous plate with material parameters is presented in Table 3 . It is obvious that an increase in the dimensional exponential index $n$ results in a decrease in the skin friction while for the increase of the Grashof numbers $G$ and $G m$, the skin friction on the porous plate shows an increasing nature.

Lastly, the variation of surface heat transfer with the suction velocity parameter $A$ for several values of Prandtl number is presented in Table 3 . Analytical results show that for given flow and material parameter, the surface heat transfer from the porous plate tends to decrease slightly by increasing the magnitude of the Prandtl number.

\section{CONCLUSION}

The governing equations for an unsteady, free convection and mass transfer flow of micropolar fluid embedded in a porous media have been studied. Analytical results are presented to illustrate the details of the flow and heat transfer characteristics and their dependence on the material parameters $G, G m, S c, \operatorname{Pr}, t$. It is observe that when the material parameters increase the streamwise velocity increases while the angular velocity, temperature field and mass transfer decreases.

\section{REFERENCES}

[1] Aero, E.L., Bulygin, A.N., Kuvshinskii, E.V. (1965). Asymmetric Hydromechanics. Journal of Applied Mathematical Mechanics. 29: 333 - 346.

[2] Ahmad, Y.B. (2010). Effects of Thermophoresis onNatural Convection boundary layer flow of a Micropolar fluid. Thermal Science 2010, 14(1): 171 181.
[3] Dep, N. V. (1968). Equations of Fluid Boundary Layer with Couple Stresses. Journal of Applied Mathematical Mechanics. 32(4): 777 - 783.

[4] Hassanien, I. A. and Essawy, A. H. (2004). Natural convection flow of micropolar fluid from a permeable uniform heat flux surface in porous media. Journal of Applied Mathematics and computation. 152(2): 323-335.

[5] Kim, Y. J. (2001). Unsteady Convection Flow of Micropolar Fluids. Acta Mechanica. 148: 105 - 116.

[6] Kim, Y. J. (2003). Transient mixed radiative convection flow of a Micropolar fluid past a moving, semi infinite vertical porous plate. International Journal of Heat and Mass transfer. 46(10): 1751-1758.

[7] Lok, Y. Y., Amin, N., Pop, I. (2006). Unsteady mixed convection flow of a Micropolar fluid near the stagnation point on a vertical surface. International Journal of Thermal Science. 45 (12): 1149 - 1157

[8] Lukaszewicz, G. (1999). Micropolar Fluids Theory and Applications. Birkhauser, Boston.

[9] Makinde,O.D. and Mhone,P.Y.(2005).Heat transfer to MHD oscillatory flow in a channel filled with porous medium. Rom.journal.phys. (50): 931-938.

[10] Mostafa,A.A. (2009). Thermal radiation effect on Unsteady MHD free convection flow past a vertical plate with temperature dependent viscosity, The Canadian Journal of Chemical Engineering.87 (1): 171 - 181

[11] Soundalgekar, V. M., Takhar, H. S. (1977). MHD Forced and Free Convective Flow Past a Semi-Infinitive Plate. AIAA, J.V. (15): 457 - 458.

[12] Takher, H. S. and Agarwal, R. S. (1998). Mixed Convective flow of a steady incompressible fluid over a stretching sheet. Journal of Research of the National Institute of Standards and Technology. 100(4): 449.

[13] Uwanta,I.J.(2002). Micropolar fluid flow in a channel with Poiseuille effects. ASSETS Series B, 1 (2): 9-23.

[14] Uwanta, I.J. (2008).Effects of mass transfer on laminar convective hydromagnetic flow of radiating gas in a vertical infinite channel. Proceedings of the August 2008 Annual National Conference of Mathematical Association of Nigeria. $89-97$ 